\title{
APLICAÇÃO DE NANOFLUIDOS DE CARBONATO DE CÁLCIO E SÍLICA NA RECUPERAÇÃO AVANÇADA DE PETRÓLEO
}

\author{
G. JAVORNIK ${ }^{1}$, J. P. PIZOLLO ${ }^{1}$, C. SOARES ${ }^{1}$, H. J. JOSÉ ${ }^{1}$ e R. F. P. M. MOREIRA ${ }^{1}$ \\ ${ }^{1}$ Universidade Federal de Santa Catarina, Departamento de Engenharia Química e \\ Engenharia de Alimentos \\ E-mail para contato: regina@enq.ufsc.br
}

\begin{abstract}
RESUMO - A recuperação avançada de petróleo (EOR) é um desafio para as diversas áreas de pesquisa e a nanotecnologia pode, potencialmente, alterar seus mecanismos e processos. Neste trabalho é apresentado um estudo experimental utilizando nanofluidos para EOR, baseado em suspensões salinas do surfactante dodecil benzeno sulfonato de sódio (SDBS) em diferentes concentrações $(5,94$ a $46,30 \mathrm{mg} / \mathrm{L}$ ) contendo $30 \mathrm{mg} / \mathrm{L}$ de nanopartículas (NP) de carbonato de cálcio e dióxido de silício. As características do nanofluido foram avaliadas visando sua aplicação na recuperação avançada do petróleo. Observou-se que tanto as nanopartículas de carbonato de cálcio quanto as de dióxido de silício adsorveram o SDBS. Nenhuma mudança significativa na viscosidade e na molhabilidade da solução de SDBS foi observada com a adição de nanopartículas na concentração de $30 \mathrm{mg} / \mathrm{L}$. Os ensaios de retenção e mobilidade em coluna de leito fixo com meio poroso mostraram que as nanopartículas obtiveram altos índices de retenção após $2 \mathrm{~h}$ de ensaio. A adição de NP à solução salina causou uma significativa recuperação incremental de petróleo, obtendo-se índices de $2,75 \%$ e 3,78 \% com o uso de soluções contendo $\mathrm{CaCO}_{3}$ e $\mathrm{SiO}_{2}$, respectivamente.
\end{abstract}

\section{INTRODUÇÃ̃O}

A recuperação avançada de petróleo (EOR) é um tema desafiador no campo da pesquisa científica. Considerando-se que o óleo facilmente recuperável está se esgotando e que muito petróleo permanece no reservatório após a aplicação de métodos convencionais, a utilização de EOR é crucial para garantir um fornecimento contínuo (Werner et al., 2011).

A recuperação primária de óleo utiliza a energia natural do reservatório, e a produção é limitada a cerca de $15 \%$ do óleo que está no reservatório. A recuperação secundária, com introdução de água no reservatório, aumenta a recuperação para cerca de $30 \%$ do óleo que está no reservatório. Após a inundação com água, ainda resta cerca de $60 \%$ de óleo nos poros do reservatório devido à alta pressão capilar da água. Uma maneira de recuperar o óleo residual é por meio da redução da tensão interfacial entre o óleo e a água pelo uso de surfactantes, que faz o óleo coalescer e fluir para fora do reservatório para os poços de produção (Emegwalu, 2009). A inundação com surfactante tem maior potencial de aumentar a recuperação de óleo do que com o uso de água (método convencional). A disponibilidade de um grande número de tensoativos torna possível a realização de um estudo sistemático da 
relação entre a estrutura do surfactante e da sua eficácia para a recuperação de óleo (Hirasaki et al., 2004). Muitos estudos com o uso de surfactantes aniônicos na recuperação avançada de petróleo foram realizados utilizando o surfactante dodecil sulfato de sódio (SDS). No entanto, apenas alguns trabalhos utilizando o dodecill benzeno sulfonato de sódio (SDBS) podem ser encontrados na literatura. O SDBS é um surfactante aniônico com maior poder de diminuição da tensão superficial e atinge a CMC em concentrações menores do que o SDS. Segundo Chagas et al. (2005), para atingir um valor de tensão superficial de $36 \mathrm{mN} / \mathrm{m}$ com o SDS é necessária uma concentração de $2800 \mathrm{mg} / \mathrm{L}$. Zhu et al. (2012) mostraram que, para o SDBS, concentração de $620 \mathrm{mg} / \mathrm{L}$ já é suficiente para se obter uma tensão superficial de $35,1 \mathrm{mN} / \mathrm{m}$.

Recentemente, a recuperação de petróleo utilizando nanofluidos tem sido reportada como um método melhorado de recuperação avançada. Voltatoni (2012) demonstrou que é possível utilizar nanopartículas que tenham a capacidade de se fixar nas rochas dos reservatórios, causando o desprendimento do petróleo, ou aprisionando as moléculas de hidrocarboneto de forma a arrastá-las para a superfície. Essas aplicações são possíveis devido ao fato de que as nanopartículas podem alterar a molhabilidade da rocha como resultado da adsorção nas paredes porosas. Até o momento, não são encontrados estudos sistemáticos utilizando nanopartículas de $\mathrm{CaCO}_{3}$ e $\mathrm{SiO}_{2}$ em conjunto com o surfactante aniônico SDBS. Sendo assim, o objetivo deste trabalho foi investigar o processo de recuperação avançada de petróleo utilizando nanopartículas de carbonato de cálcio $\left(\mathrm{CaCO}_{3}\right)$ e dióxido de silício $\left(\mathrm{SiO}_{2}\right)$ em soluções aquosas de dodecil benzeno (SDBS).

\section{MATERIAIS E MÉTODOS}

\subsection{Materiais}

Foram utilizados o surfactante aniônico dodecil benzeno sulfonato de sódio (SDBS) da empresa Sigma Aldrich, nanopartículas de carbonato de cálcio $\left(\mathrm{CaCO}_{3}\right)$ e sílica $\left(\mathrm{SiO}_{2}\right)$ adquiridas da empresa SSNano. Todos os experimentos foram realizados com água salina cuja formulação foi fornecida pela Petrobras.

\subsection{Métodos}

Preparo das soluções para os testes de adsorção de SDBS sobre as nanopartículas: foram preparadas soluções de SDBS em concentrações de 5,94; 9,78; 13,50; 17,50; 22,62; 35,30 e $46,30 \mathrm{mg} / \mathrm{L}$. Em seguida, a essa solução foram adicionados $30 \mathrm{mg} / \mathrm{L}$ de $\mathrm{CaCO}_{3}$ ou $\mathrm{SiO}_{2}$. As suspensões eram mantidas sob agitação em um jar test e, em intervalos de tempo de contato de 24,48 e 72 horas, eram retiradas alíquotas, que eram subsequentemente centrifugadas a $1500 \mathrm{rpm}$.

Caracterização dos nanofluidos: para caracterização dos nanofluidos foram realizadas análises de tensão superficial (método da placa de Wilhelmy), ângulo de contato (goniômetro - Contact Angle Tool), viscosidade (reômetro) e carbono orgânico total (COT). A massa adsorvida $(q)$ foi calculada utilizando a Equação 1:

$$
q=\frac{V\left(C_{0}-C_{e}\right)}{m}
$$


onde $C_{0}$ é a concentração inicial de tensoativo $(\mathrm{mg} / \mathrm{L}), C_{e}$ é a concentração de tensoativo no equilíbrio (mg/L), $V$ é o volume da solução (L) e $m$ é a massa do adsorvente (mg).

Montagem da coluna de leito fixo: os testes de deslocamento de óleo e de retenção e mobilidade das NP em meio poroso foram realizados em uma coluna de leito fixo utilizando areia como meio poroso.

Mobilidade e retenção de NP: nos ensaios de retenção e mobilidade foi utilizada suspensão aquosa contendo $5 \mathrm{mg} / \mathrm{L}$ de nanopartículas $\left(\mathrm{CaCO}_{3}\right.$ ou $\left.\mathrm{SiO}_{2}\right)$, agitada constantemente, que percolava a coluna em sentido descendente, à vazão de $30 \mathrm{~mL} / \mathrm{min}$ com auxílio de uma bomba peristáltica. A quantificação da concentração de saída das nanopartículas foi feita com análises de absorção atômica. $\mathrm{O}$ experimento foi realizado a $25^{\circ} \mathrm{C}$.

Determinação da remoção de petróleo no meio poroso: para determinação da remoção de petróleo, foi utilizada a coluna de leito fixo com areia impregnada de petróleo. Estes ensaios foram realizados em três etapas. Primeiramente, a recuperação secundária foi realizada pela alimentação de água salina até que não houvesse mais petróleo na saída da coluna. Em seguida, realizou-se a recuperação avançada, pela alimentação de uma solução de SDBS (46,3 mg/L) em água salina, até que não houvesse petróleo na saída da coluna. E, por fim, novamente a recuperação avançada foi realizada, agora pela alimentação de suspensão do nanofluido, sendo que a formulação do nanofluido era composta de nanopartículas de $\mathrm{CaCO}_{3}$

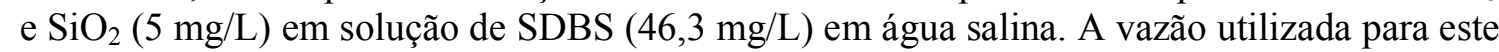
ensaio foi de $3 \mathrm{~mL} / \mathrm{min}$. A determinação do óleo recuperado era realizada gravimetricamente.

\section{RESULTADOS E DISCUSSÃO}

\subsection{Características da suspensão de nanopartículas em solução salina de SDBS}

Tensão superficial: a Tabela 1 apresenta os valores de tensão superficial inicial e após $72 \mathrm{~h}$ de contato das $\mathrm{NP}$ de $\mathrm{CaCO}_{3}$ e $\mathrm{SiO}_{2}$ com a solução salina de SDBS. Observa-se que, com exceção da menor concentração de SDBS, todas soluções apresentaram um leve aumento da tensão superficial após $72 \mathrm{~h}$ de contato com as NP. Os resultados indicam que pode ocorrer a adsorção do $\mathrm{SDBS}$ nas $\mathrm{NP}$ de $\mathrm{SiO}_{2}$ e $\mathrm{CaCO}_{3}$, resultando na diminuição da concentração de SDBS na fase aquosa e, consequentemente, no aumento da tensão superficial do nanofluido.

Carbono Orgânico Total (COT): a Tabela 2 apresenta os resultados de COT de soluções de SDBS antes e após o contato com NP de $\mathrm{CaCO}_{3}$ e $\mathrm{SiO}_{2}$. Observa-se que, tanto para o $\mathrm{CaCO}_{3}$ como para $\mathrm{SiO}_{2}$, a partir da concentração $9,78 \mathrm{mg} / \mathrm{L}$ de SDBS, houve um decréscimo nos valores de COT já nas primeiras $24 \mathrm{~h}$ de contato, comprovando a adsorção do SDBS na superfície do sólido, o que justifica os resultados mostrados anteriormente da tensão superficial. 
Tabela 1 - Valores de tensão superficial inicial e após 72 h de contato para a suspensão de $\mathrm{CaCO}_{3}$ e $\mathrm{SiO}_{2}$ em solução salina de SDBS

\begin{tabular}{lccccccc}
\hline \multicolumn{1}{c}{ Concentração (mg/L) } & \multicolumn{5}{c}{ Tensão superficial (mN/m) } \\
\hline \multirow{2}{*}{ SDBS Nanopartículas } & $\begin{array}{c}\text { Solução } \\
\text { SDBS }\end{array}$ & \multicolumn{5}{c}{ Solução SDBS com adição de NP } \\
\cline { 3 - 8 } & & $\begin{array}{c}\text { Desvio } \\
\text { padrão }\end{array}$ & $\mathrm{CaCO}_{3}$ & $\begin{array}{c}\text { Desvio } \\
\text { padrão }\end{array}$ & $\mathrm{SiO}_{2}$ & $\begin{array}{c}\text { Desvio } \\
\text { padrão }\end{array}$ \\
\hline 5,94 & 30 & 36,80 & $\pm 0,44$ & 36,85 & $\pm 0,83$ & 36,51 & $\pm 0,48$ \\
9,78 & 30 & 34,55 & $\pm 0,29$ & 33,98 & $\pm 0,23$ & 35,14 & $\pm 0,29$ \\
13,50 & 30 & 31,11 & $\pm 0,23$ & 31,60 & $\pm 0,82$ & 35,51 & $\pm 0,35$ \\
17,50 & 30 & 31,78 & $\pm 0,18$ & 33,01 & $\pm 0,25$ & 37,53 & $\pm 1,24$ \\
22,62 & 30 & 31,85 & $\pm 0,30$ & 33,98 & $\pm 0,65$ & 32,94 & $\pm 0,24$ \\
35,30 & 30 & 30,60 & $\pm 0,48$ & 33,44 & $\pm 0,45$ & 32,35 & $\pm 0,38$ \\
46,30 & 30 & 29,18 & $\pm 0,22$ & 31,35 & $\pm 0,23$ & 31,73 & $\pm 0,16$ \\
\hline
\end{tabular}

Tabela 2 - Concentração de Carbono Orgânico Total da solução de SDBS antes e após o contato com nanopartículas de (a) $\mathrm{CaCO}_{3}$ e (b) $\mathrm{SiO}_{2}$

\begin{tabular}{|c|c|c|c|c|c|c|c|c|}
\hline \multirow{3}{*}{$\begin{array}{c}\text { (a) } \\
\mathrm{C}_{\mathrm{SDBS}} \\
(\mathrm{mg} / \mathrm{L})\end{array}$} & \multicolumn{8}{|c|}{ Concentração de Carbono Orgânico total, mg/L } \\
\hline & \multirow{2}{*}{ Inicial } & \multirow{2}{*}{$\begin{array}{l}\text { Desvio } \\
\text { padrão }\end{array}$} & \multicolumn{6}{|c|}{$\mathrm{CaCO}_{3}$} \\
\hline & & & $24 \mathrm{~h}$ & $\begin{array}{l}\text { Desvio } \\
\text { padrão }\end{array}$ & $48 \mathrm{~h}$ & $\begin{array}{l}\text { Desvio } \\
\text { padrão }\end{array}$ & $72 \mathrm{~h}$ & $\begin{array}{l}\text { Desvio } \\
\text { padrão }\end{array}$ \\
\hline 5,94 & 4,93 & $\pm 0,10$ & 4,00 & $\pm 0,08$ & 4,61 & $\pm 0,26$ & - & $\pm 0,05$ \\
\hline 9,78 & 8,41 & $\pm 0,07$ & 6,71 & $\pm 0,12$ & 6,12 & $\pm 0,10$ & 5,97 & $\pm 0,20$ \\
\hline 13,50 & 9,03 & $\pm 0,08$ & 8,03 & $\pm 0,05$ & 7,90 & $\pm 0,21$ & 7,65 & $\pm 0,20$ \\
\hline 17,50 & 10,20 & $\pm 0,19$ & 8,93 & $\pm 0,92$ & 8,81 & $\pm 0,14$ & 8,59 & $\pm 0,12$ \\
\hline 22,62 & 14,04 & $\pm 0,36$ & 10,54 & $\pm 1,16$ & 10,35 & $\pm 0,31$ & 11,55 & $\pm 0,37$ \\
\hline 35,30 & 21,91 & $\pm 0,68$ & 10,17 & $\pm 0,12$ & - & - & 9,73 & $\pm 0,28$ \\
\hline 46,30 & 35,48 & $\pm 0,34$ & - & - & 8,50 & $\pm 0,20$ & 7,40 & $\pm 0,31$ \\
\hline \multirow{3}{*}{$\begin{array}{c}\text { (b) } \\
\mathrm{C}_{\mathrm{SDBS}} \\
(\mathrm{mg} / \mathrm{L})\end{array}$} & \multicolumn{8}{|c|}{ Concentração de Carbono Orgânico total, mg/L } \\
\hline & \multirow[b]{2}{*}{ Inicial } & \multirow{2}{*}{$\begin{array}{l}\text { Desvio } \\
\text { padrão }\end{array}$} & \multicolumn{6}{|c|}{$\mathrm{SiO}_{2}$} \\
\hline & & & $24 \mathrm{~h}$ & $\begin{array}{l}\text { Desvio } \\
\text { padrão }\end{array}$ & $48 \mathrm{~h}$ & $\begin{array}{l}\text { Desvio } \\
\text { padrão }\end{array}$ & $72 \mathrm{~h}$ & $\begin{array}{l}\text { Desvio } \\
\text { padrão }\end{array}$ \\
\hline 5,94 & 4,93 & $\pm 0,10$ & 4,99 & $\pm 0,11$ & 4,79 & $\pm 0,21$ & 4,62 & $\pm 0,13$ \\
\hline 9,78 & 8,41 & $\pm 0,07$ & 7,24 & $\pm 0,17$ & 6,52 & $\pm 0,22$ & 6,56 & $\pm 0,21$ \\
\hline 13,50 & 9,03 & $\pm 0,08$ & 7,00 & $\pm 1,33$ & 7,07 & $\pm 0,08$ & 6,89 & $\pm 0,08$ \\
\hline 17,50 & 10,20 & $\pm 0,19$ & 7,58 & $\pm 0,99$ & 6,58 & $\pm 0,20$ & 6,81 & $\pm 0,15$ \\
\hline 22,62 & 14,04 & $\pm 0,36$ & 16,47 & $\pm 0,18$ & 12,05 & $\pm 0,18$ & 9,39 & $\pm 0,26$ \\
\hline 35,30 & 21,91 & $\pm 0,68$ & 16,15 & $\pm 0,25$ & 13,41 & $\pm 1,67$ & 11,01 & $\pm 0,10$ \\
\hline 46,30 & 35,48 & $\pm 0,34$ & 7,38 & $\pm 0,14$ & 7,36 & $\pm 0,78$ & 6,94 & $\pm 0,07$ \\
\hline
\end{tabular}


Com estes resultados foi possível calcular a massa adsorvida de SDBS nas nanopartículas de $\mathrm{CaCO}_{3}$ e $\mathrm{SiO}_{2}$ (Tabela 3). Os resultados mostrados na Tabela 3 indicam que o SDBS é adsorvido nas nanopartículas hidrofóbicas de sílica e carbonato de cálcio.

Tabela 3 - Concentração inicial, concentração de equilíbrio e quantidade adsorvida de SDBS com adição de $\mathrm{CaCO}_{3}$ ou $\mathrm{SiO}_{2}{ }^{*}$

\begin{tabular}{ccccc}
\hline \multirow{2}{*}{\begin{tabular}{c}
$\mathrm{C}_{\text {SDBS inicial }}(\mathrm{mg} / \mathrm{L})$ \\
\cline { 2 - 5 }
\end{tabular}} & $\begin{array}{c}\mathrm{C}_{\text {SDBS }}(\mathrm{mg} / \mathrm{L}) \text { após } \\
\text { adsorção com } \mathrm{CaCO}_{3}\end{array}$ & $\begin{array}{c}\text { Massa } \\
\text { adsorvida, } \\
\mathrm{mg} / \mathrm{g}\end{array}$ & $\begin{array}{c}\mathrm{C}_{\text {SDBS }}(\mathrm{mg} / \mathrm{L}) \text { após } \\
\text { adsorção com } \mathrm{SiO}_{2}\end{array}$ & $\begin{array}{c}\text { Massa } \\
\text { adsorvida, } \\
\mathrm{mg} / \mathrm{g}\end{array}$ \\
\hline \multicolumn{2}{c}{$\mathrm{CaCO}_{3}$} & & \multicolumn{3}{c}{$\mathrm{SiO}_{2}$} & \\
9,78 & 5,18 & 25,28 & 5,77 & 5,51 \\
13,50 & 7,29 & 82,97 & 7,88 & 63,37 \\
17,50 & 11,76 & 58,14 & 10,45 & 101,57 \\
22,62 & 15,06 & 81,50 & 11,99 & 183,60 \\
35,30 & 17,42 & 173,28 & 15,13 & 249,72 \\
46,30 & 15,68 & 654,11 & 17,74 & 585,37 \\
\hline
\end{tabular}

* Volume de solução salina: 1 litro; massa de NP: $30 \mathrm{mg}$; temperatura: $25^{\circ} \mathrm{C}$

Viscosidade dos nanofluidos: a dependência da tensão de cisalhamento com a taxa de cisalhamento e a dependência da viscosidade com a taxa de cisalhamento para a solução de SDBS 46,30 mg/L com e sem adição de nanopartículas de $\mathrm{CaCO}_{3}$ e $\mathrm{SiO}_{2}$ são apresentadas na Figura 1.

Pode-se observar que a solução de SDBS em água salina, tanto na presença de nanopartículas de sílica quanto de carbonato de cálcio, apresenta um comportamento típico de fluido newtoniano. Todas as concentrações de SDBS testadas apresentaram esse mesmo comportamento. Estes resultados indicam que a presença das nanopartículas de sílica ou carbonato de cálcio não altera a viscosidade do fluido.

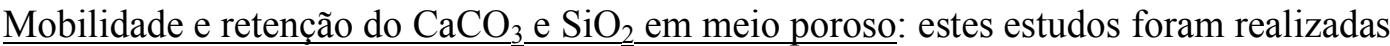
para observar o comportamento das NP no deslocamento em coluna de leito fixo, principalmente em relação à retenção e mobilidade destas. Os experimentos foram realizados sem adição de SDBS e as análises de absorção atômica mostraram que houve retenção quase que total para ambas as nanopartículas com índices de retenção de $92 \%$ para o carbonato de cálcio e $85 \%$ para a sílica (Tabela 4 ).

Determinação da molhabilidade e ângulo de contato: na Tabela 5 encontram-se os valores de ângulo de contato com e sem adição de $\mathrm{CaCO}_{3}$ e $\mathrm{SiO}_{2}$. Observa-se que nas amostras sem adição de nanopartículas, os valores de ângulo de contato não apresentaram variação significativa, mesmo com o aumento da concentração de SDBS. E, também, a adição das nanopartículas não acarretou aumento significativo do ângulo de contato dos nanofluidos em relação à superfície sólida. 


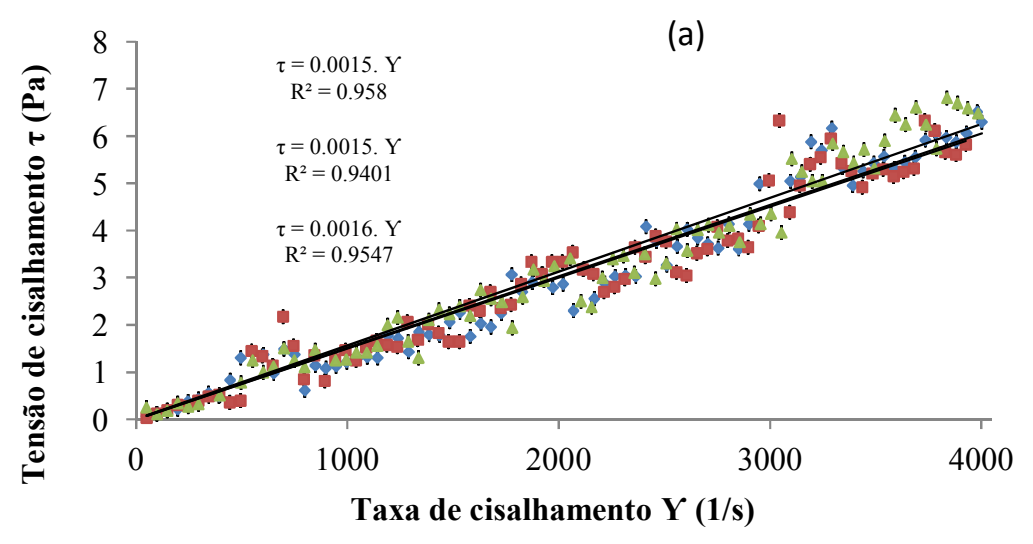

- Sem adição de NP • Com adição de $\mathrm{CaCO} 3$ • Com adição de SiO2

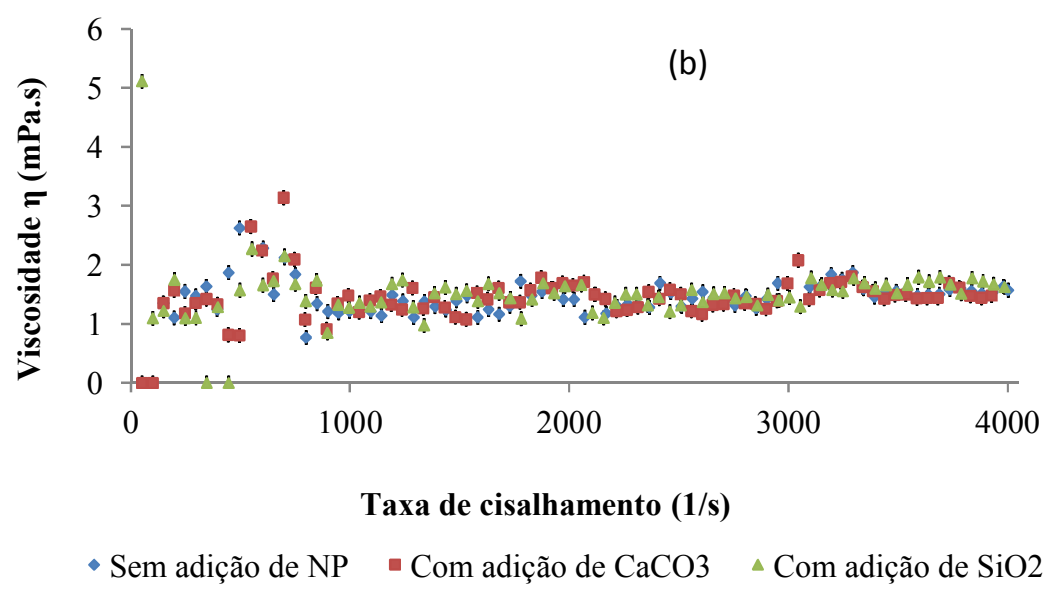

Figura 1 - Dependência da tensão de cisalhamento (a) e da viscosidade (b) com a taxa de cisalhamento para a solução de SDBS 46,30 mg/L em água salina com e sem adição de NP.

Tabela 4 - Valores de retenção das nanopartículas de carbonato de cálcio e sílica

\begin{tabular}{cc}
\hline Nanopartícula & Retenção $(\%)$ \\
\hline Carbonato de cálcio & 92 \\
Ś́lica & 85 \\
\hline
\end{tabular}

Recuperação de petróleo utilizando os nanofluidos: os nanofluidos de $\mathrm{CaCO}_{3}$ e $\mathrm{SiO}_{2}$ foram utilizados como meio de recuperação terciária de petróleo. Os resultados de recuperação são apresentados na Tabela 6. Para os nanofluidos com NP de $\mathrm{CaCO}_{3}$ ou $\mathrm{SiO}_{2}$, a recuperação incremental foi de $2,75 \%$ e $3,78 \%$, respectivamente. 
Tabela 5 - Ângulos de contato de soluções de SDBS em água do mar com e sem adição de $\mathrm{CaCO}_{3}$ e $\mathrm{SiO}_{2}$. Medidas realizadas sobre placa de vidro

\begin{tabular}{ccccccc}
\hline & \multicolumn{5}{c}{ Ângulo de contato $\left(^{\circ}\right)$} \\
\cline { 2 - 6 } $\begin{array}{c}\mathrm{C}_{\text {SDBS }} \\
(\mathrm{mg} / \mathrm{L})\end{array}$ & $\begin{array}{c}\text { Sem adição } \\
\text { de NP }\end{array}$ & $\begin{array}{c}\text { Desvio } \\
\text { padrão }\end{array}$ & $\begin{array}{c}\text { Com adição } \\
\text { de } \mathrm{CaCO}_{3}\end{array}$ & $\begin{array}{c}\text { Desvio } \\
\text { padrão }\end{array}$ & $\begin{array}{c}\text { Com adição } \\
\text { de } \mathrm{SiO}_{2}\end{array}$ & $\begin{array}{c}\text { Desvio } \\
\text { padrão }\end{array}$ \\
\hline 5,94 & 57,06 & $\pm 0,14$ & 62,09 & $\pm 0,34$ & 64,51 & $\pm 2,56$ \\
9,78 & 57,15 & $\pm 1,41$ & 60,55 & $\pm 0,32$ & 63,06 & $\pm 2,85$ \\
13,50 & 55,94 & $\pm 0,91$ & 60,15 & $\pm 0,74$ & 62,20 & $\pm 2,73$ \\
17,50 & 57,91 & $\pm 1,11$ & 60,70 & $\pm 0,10$ & 57,84 & $\pm 0,71$ \\
22,62 & 56,32 & $\pm 1,37$ & 61,85 & $\pm 0,67$ & 58,04 & $\pm 0,21$ \\
35,30 & 57,03 & $\pm 1,12$ & 63,46 & $\pm 0,16$ & 59,68 & $\pm 2,42$ \\
46,30 & 56,42 & $\pm 0,64$ & 63,08 & $\pm 0,10$ & 58,94 & $\pm 0,25$ \\
\hline
\end{tabular}

Tabela 6 - Recuperação de petróleo utilizando o método secundário e avançado

\begin{tabular}{ccc}
\hline & \multicolumn{2}{c}{ Recuperação (\%) } \\
\cline { 2 - 3 } & $\mathrm{CaCO}_{3}$ & $\mathrm{SiO}_{2}$ \\
\hline Água salgada & 28,65 & 36,10 \\
Água salgada + SDBS & 1,42 & 0,73 \\
Água salgada + SDBS + NP & 2,75 & 3,78 \\
Recuperação total & 32,82 & 39,95 \\
\hline
\end{tabular}

Hendraningrat et al. (2012) estudaram a recuperação de petróleo utilizando inundação com nanofluidos em recuperação terciária. As nanopartículas utilizadas foram dióxido de silício $\left(\mathrm{SiO}_{2}\right)$, óxido de alumínio $\left(\mathrm{Al}_{2} \mathrm{O}_{3}\right)$, dióxido de titânio $\left(\mathrm{TiO}_{2}\right)$ e óxido de ferro $\left(\mathrm{Fe}_{2} \mathrm{O}_{3}\right)$ em concentrações de 0,01 e $0,05 \%$ em massa. Após os ensaios, os autores conseguiram uma recuperação de petróleo inferior a $2 \%$. Segundo estes autores, ao se injetarem nanopartículas em solução, estas podem se fixar no interior de uma película de água entre a superfície e uma gotícula de óleo executando uma pressão adicional entre a gotícula de óleo e a película de água, separando as duas fases. Este fenômeno poderia explicar a mobilização de óleo que ocorreu neste trabalho durante a inundação terciária com nanopartículas. Cabe salientar que a concentração utilizada de NP utilizada no presente trabalho foi de $5 \mathrm{mg} / \mathrm{L}$, muito menor que a utilizada por Hendraningrat et al. (2012) (100 mg/L) e, ainda assim, foi possível se obter índices de recuperação de petróleo semelhantes.

Segundo Giraldo et al. (2013), surfactantes induzem à alteração da molhabilidade. Argumenta-se que, uma vez que moléculas de óleo são removidas pelo surfactante da superfície da rocha, estes podem ser adsorvidas na superfície limpa. Se nanopartículas com alta afinidade pelo óleo são adicionadas ao surfactante, o desempenho do tratamento pode ser melhorado. Estas NP serão facilmente adsorvidas em superfícies cobertas por uma fase óleo e podem contribuir para a durabilidade do tratamento. Também, as NP podem evitar uma posterior agregação das moléculas de óleo que foram removidas, mantendo-as em suspensão. Isto explicaria o motivo da recuperação avançada de petróleo ter sido maior com $\mathrm{SiO}_{2}$ hidrofóbico, ou seja, com alta afinidade pelo óleo. 


\section{9 a 22 de outubro de 2014 \\ Florianópolis/SC}

\section{CONCLUSÃO}

A adição das nanopartículas de carbonato de cálcio e sílica à solução salina de SDBS teve efeito significativo para a recuperação avançada de petróleo, atingindo índices de recuperação incremental de 2,75 e 3,78 \%, respectivamente. Apesar dos resultados de tensão superficial, molhabilidade e viscosidade divergirem dos reportados na literatura, houve recuperação incremental de petróleo corroborando com a hipótese de Hendraningrat e colaboradores de que, ao se injetarem nanopartículas em solução, estas podem se fixar no interior de uma película de água entre a superfície e uma gotícula de óleo executando uma pressão adicional entre a gotícula de óleo e a película de água separando as duas fases.

\section{REFERÊNCIAS}

CHAGAS, E. F.; VALENTIM, A. C. M.; GARCIA, R. B. Avaliação da tensão superficial e comportamento reológico de um complexo polimérico para restauração de poços de petróleo. $3^{\circ}$ Congresso Brasileiro de P\&D em Petróleo e Gás, Salvador, outubro, 2005.

EMEGWALU, C. C. Enhanced oil recovery: surfactant flooding as a possibility for the norne e-segment. Reservoir engineering specialization project report. Department of Petroleum Engineering and Applied Geophysics. Norwegian University of Science and Technology, 2009. Disponível em http://www.ipt.ntnu.no/ norne/wiki/data/media/english/thesis/chinenyeclaraemegwalu.p df. Acessado em: setembro, 2013.

GIRALDO, J.; BENJUMEA, P.; LOPERA, S. CORTÉS, F. B.; RUIZ, M. A. Wettability alteration of sandstone cores by alumina-based nanofluids. Energy Fuels, v. 27, p. 3659-3665, 2013.

HENDRANINGRAT, L.; ENGESET, B.; SUWARNO, S.; TORSAETER, O. Improved oil recovery by nanofluids flooding: an experimental study. SPE, Norwegian University of Science and Technology (NTNU), SPE 163335, December, 2012.

HIRASAKI, G. J.; MILlER, C. A.; POPE, G. A.; JACKSON, R. E. Surfactant based enhanced oil recovery and foam mobility control. $1^{\text {st }}$ Annual Technical Report, Rice University, The University of Texas, INTERA, Inc. February, 2004. Disponível em: http://www.netl.doe.gov/KMD/cds/disk37/E\%20-

\%20PRIME\%20Program/15406\%201st\%20semi-annual\%202003.pdf. Acessado em: setembro, 2013.

WEVER, D. A. Z., PICCHIONI F., BROEKHUIS, A. A. Polymers for enhanced oil recovery: a paradigm forstructure - property relationship in aqueous solution. Prog. Polym. Sci., v. 36, p. $1558-1628,2011$.

VOLTATONI, T. Emprego de ciclodextinas para a recuperação avançada de petróleo. Santo André, SP, 2012. Dissertação, Universidade Federal do ABC - Centro de Engenharias, Modelagem e Ciências Sociais Aplicadas - Curso de Pós Graduação em Energia, Santo André, 2012.

ZHU, D. Y.; CHENGA, F.; CHENA, Y.; JIANG, S. C. Preparation, characterization and properties of anionic gemini surfactants with long rigid or semi-rigid spacers. Colloid. Surface. A, v. 397, p. 1-7, 2012. 